\title{
Direct cholangioscopy for diagnosis and treatment guidance of suspected biliary papillomatosis with malignant transformation
}

A 48-year-old man with no significant past medical history was admitted with jaundice and abdominal pain. Total bilirubin was $6.5 \mathrm{mg} / \mathrm{dL}$. The magnetic resonance cholangiopancreatography demonstrated intra- and extrahepatic bile duct dilation secondary to a stricture in the mid common bile duct (CBD) and a parietal thickening extending from the mid-CBD stricture to the hilum, and left and right hepatic ducts ( $\triangleright$ Fig. 1 ). Biliary papillomatosis was suspected, with possible malignant transformation. On imaging, foramen of Winslow adenopathy was suspicious for malignant involvement.

The patient underwent an endoscopic retrograde cholangiopancreatography with direct retrograde cholangioscopy for proper characterization of the lesions, evaluation of their extent, and targeted biopsies ( $\downarrow$ Video 1 ). The aspect of the CBD stricture was worrisome with an irregular pattern and vascularity ( $>$ Fig. 2 ), suggesting cholangiocarcinoma. A polypoid, papillary pattern was noted as well, from above the stricture up to the left and right hepatic ducts ( 3 and $4 \mathrm{~cm}$ proximal to the hilum, respectively) ( $\mathbf{F i g . 3 )}$. Targeted biopsies of the stricture and the papillary lesion were taken, and a plastic stent was placed across the stricture.

Pathology confirmed cholangiocarcinoma at the level of the stricture, and biliary papillomatosis at the level of the papillary lesion.

Surgical resection focusing on the tumorinvolved bile duct (i.e. extrahepatic) was decided, with resection of the extrahepatic bile duct, lymphadenectomy, and hepaticojejunostomy. Final staging was
pT2N1M0 (R1). The patient is presently undergoing adjuvant chemotherapy.

Biliary papillomatosis is a rare papillary tumor that extends within the biliary tree and may progress to cholangiocarcinoma $[1,2]$. Dilated bile ducts and intraductal soft-tissue masses can be seen on cross-sectional imaging [3]. Cholangioscopy can help to target biopsies when cholangiocarcinoma is suspected, and allows a precise assessment of the extent of the disease. Radical resection is warranted given the high risk of malignant transformation [4]. This case highlights how cholangioscopy may assist in therapeutic decision making and guide the surgeons to that effect.

Endoscopy_UCTN_Code_CCL_1AZ_2AC
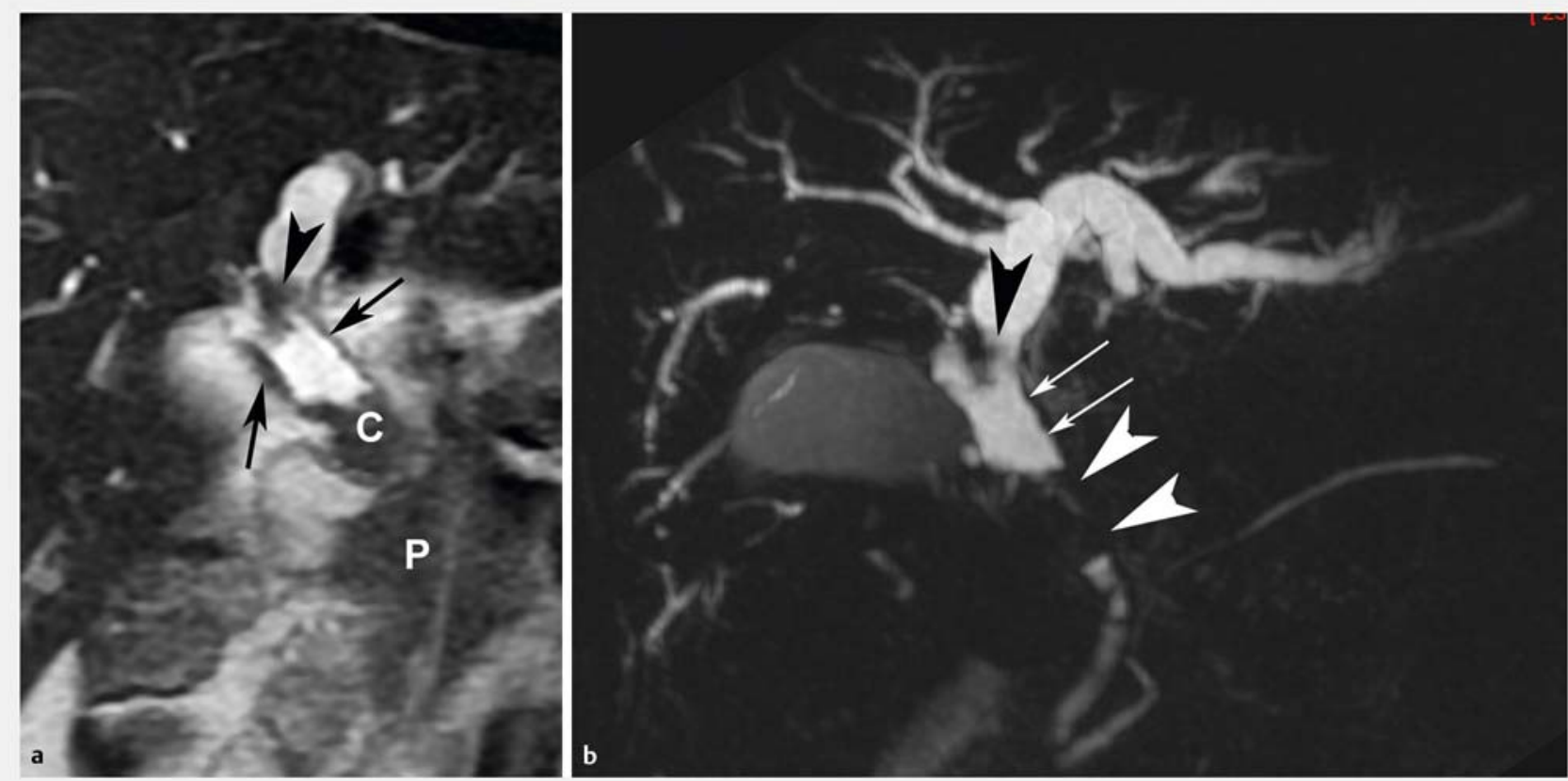

- Fig. 1 Magnetic resonance cholangiography. a Coronal T2-weighted image. C, stricture in the mid common bile duct (CBD), suspicious for cholangiocarcinoma. P, superior part of the pancreas. Black arrowhead, left hepatic duct stricture, suspicious for cholangiocarcinoma; black arrows, parietal thickening of the CBD, consistent with biliary papillomatosis. b Maximum intensity projection reconstruction. White arrowheads, tight CBD stricture related to cholangiocarcinoma; white arrows, bile duct wall irregularities related to biliary papillomatosis; black arrowhead, left hepatic duct stricture, suspicious for cholangiocarcinoma. 


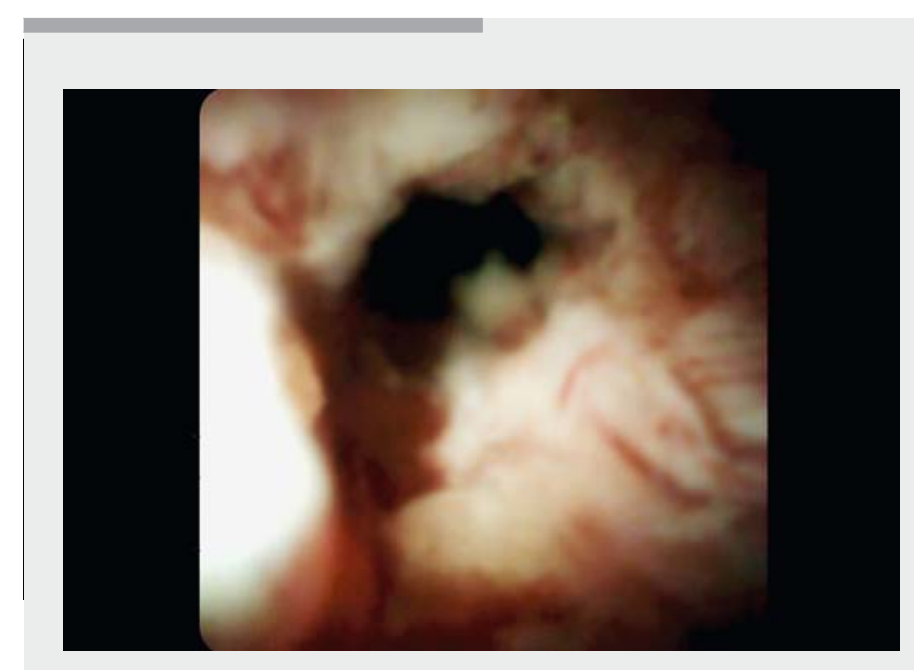

Video 1 Cholangioscopy showing a stricture in the mid common bile duct (CBD) with features consistent with cholangiocarcinoma, as well as a widespread papillary intraductal soft tissue mass involving the CBD, hilum, and left and right hepatic ducts.
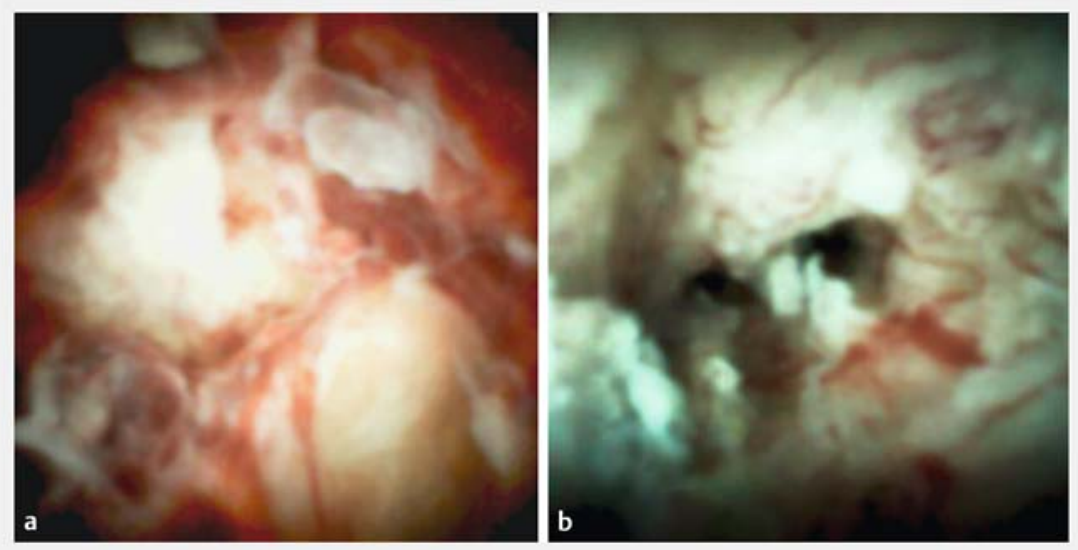

- Fig. 2 Cholangioscopy. a, b Stricture in the mid common bile duct with an irregular vascularity and pattern.
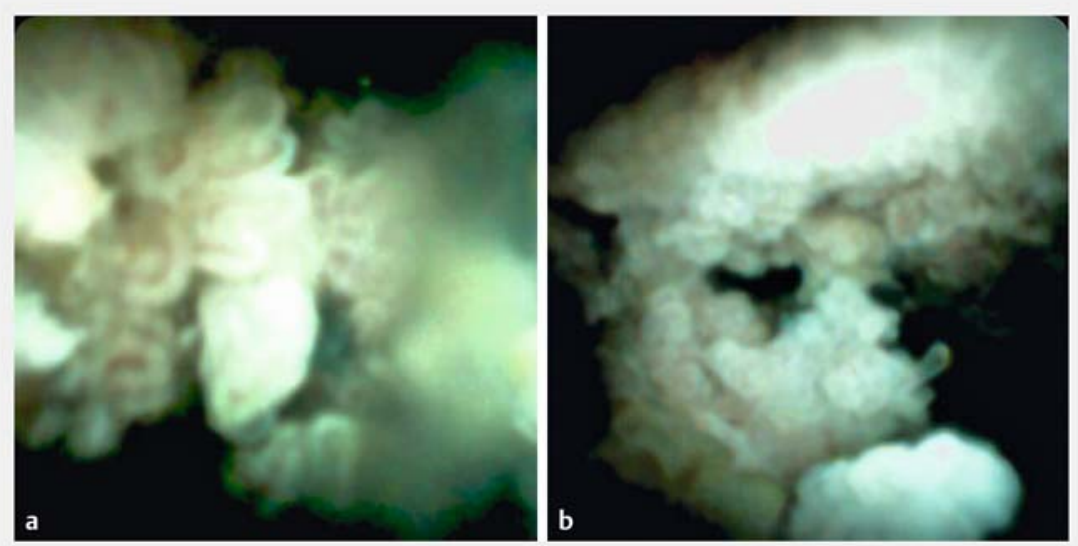

- Fig. 3 Cholangioscopy. a, b Polypoid, papillary pattern consistent with papillomatosis.

\section{Competing interests}

Drs. Camus and Chaput are consultants for Boston Scientific.

The authors

Aymeric Becq ${ }^{1,2}$, Marine Camus ${ }^{1,2}$, Olivia Pietri $^{3}$, Lionel Arrivé ${ }^{4}$, Francois Paye ${ }^{5}$, Xavier Dray $^{1,2}$, Ulriikka Chaput ${ }^{1,2}$

1 Endoscopy Department, Saint Antoine Hospital, Paris, France

2 Sorbonne University, AP-HP, Saint Antoine Hospital, Paris, France

3 Hepatology Department, Saint Antoine Hospital, Paris, France

4 Radiology Department, Saint Antoine Hospital, Paris, France

5 Digestive Surgery Department, Saint Antoine Hospital, Paris, France

\section{Corresponding author}

\section{Aymeric Becq, MD, MS}

Endoscopy Department, Saint Antoine Hospital, 184 Rue du Faubourg Saint Antoine, Paris, 75012, France

Fax: +33-1-49282970

aymeric.becq@aphp.fr

\section{References}

[1] Neumann RD, LiVolsi VA, Rosenthal NS et al. Adenocarcinoma in biliary papillomatosis. Gastroenterology 1976; 70: 779-782

[2] Lee SS, Kim M-H, Lee SK et al. Clinicopathologic review of 58 patients with biliary papillomatosis. Cancer 2004; 100: 783 - 793

[3] Hoang TV, Bluemke DA. Biliary papillomatosis: CT and MR findings. J Comput Assist Tomogr 1998; 22: 671-672

[4] Gu C, Lin Y, Jin H et al. Biliary papillomatosis with malignant transformation: a case report and review of the literature. Oncol Lett 2015; 10: 3315-3317

\section{Bibliography}

DOI https://doi.org/10.1055/a-0977-2327

Published online: 9.8.2019

Endoscopy 2020; 52: E3-E4

(c) Georg Thieme Verlag KG

Stuttgart $\cdot$ New York

ISSN 0013-726X 